\title{
PERFIL EPIDEMIOLÓGICO DE GESTANTES DE ALTO RISCO
}

\author{
Lediana Dalla Costa ${ }^{1}$, Caroline Cales Cura ${ }^{2}$, Alessandro Rodrigues Perondi ${ }^{3}$, Vivian Francielle França ${ }^{4}$, \\ Durcelina Schiavoni Bortoloti ${ }^{5}$
}

\begin{abstract}
RESUMO: O objetivo do presente estudo foi traçar o perfil epidemiológico de gestantes de alto risco atendidas no Instituto da Mulher, Secretaria de Saúde do município de Francisco Beltrão. O estudo avaliou 61 prontuários, entre janeiro e junho de 2015. Verificou-se que $82 \%(n=50)$ das gestantes tinham de 15 a 35 anos; $47,5 \%(n=29)$ possuíam o segundo grau completo; $52,5 \%(n=32)$ casadas; $62,3 \%(n=38)$ da cor branca; $88,5 \%$ $(n=54)$ com antecedentes familiares de doença crônica, sendo 63,9\% ( $n=39)$ com hipertensão arterial; $82 \%(n=50)$ com antecedentes pessoais, somando 52,5\% ( $n=32)$ casos de infecção urinária; $70,5 \%(n=43)$ eram multíparas. A gestação de risco evoluiu para cesarianas em $80,3 \%(n=49)$ dos casos. Este estudo produziu informações importantes a respeito do perfil desta população, o que permite os profissionais envolvidos no atendimento a gestante de alto rico possa exercer um papel fundamental na redução da mortalidade materna. Conclui-se que estas gestantes de alto risco aparentemente não apresentam perfil diferente da realidade de outros municípios do Brasil, principalmente quando a hipertensão foi constatada como importante fator de antecedente familiar, pessoal e prevalência atual.
\end{abstract}

DESCRITORES: Gravidez de alto risco; Perfil de saúde; Cuidado pré-natal; Gestantes; Saúde da mulher.

\section{EPIDEMIOLOGICAL PROFILE OF HIGH-RISK PREGNANT WOMEN}

ABSTRACT: The objective in this study was to outline the epidemiological profile of high-risk pregnant women attended at the Instituto da Mulher of the Municipal Health Department in the city of Francisco Beltrão, Brazil. Sixty-one patient histories were assessed between January and June 2015. It was verified that $82 \%(\mathrm{~N}=50)$ of the pregnant women were between 15 and 35 years of age; $47.5 \%$ $(\mathrm{N}=29)$ had finished secondary education; $52.5 \%(\mathrm{~N}=32)$ were married; $62.3 \%(\mathrm{~N}=38)$ were white; $88.5 \%(\mathrm{~N}=54)$ had family antecedents of chronic illness, $63.9 \%(\mathrm{~N}=39)$ related to arterial hypertension; $82 \%(\mathrm{~N}=50)$ had personal antecedents, with $52.5 \%(\mathrm{~N}=32)$ of urinary infection cases; $70.5 \%(\mathrm{~N}=43)$ were multipara. The high-risk pregnancy evolved to caesarean sections in $80.3 \%(\mathrm{~N}=49)$ of the cases. This study produced important information about the profile of this population, which allows the professionals involved in care delivery to high-risk pregnant women to play a fundamental role in the reduction of maternal mortality. In conclusion, the profile of these high-risk pregnant women seemingly does not differ from the reality in other Brazilian cities, mainly concerning hypertension as an important factor of family and personal antecedents and current prevalence.

DESCRIPTORS: High-risk pregnancy; Health profile; Prenatal care; Pregnant women; Woman's health.

\section{PERFIL EPIDEMIOLÓGICO DE EMBARAZADAS DE ALTO RIESGO}

RESUMEN: El objetivo del presente estudio fue trazar el perfil epidemiológico de embarazadas de alto riesgo atendidas en el Instituto da Mulher, Secretaría de Salud del municipio de Francisco Beltrão. El estudio evaluó 61 archivos, entre enero y junio de 2015. Fue verificado que $82 \%(n=50)$ de las embarazadas tenían de 15 a 35 años; $47,5 \%$ (n=29) poseía el segundo grado completo; $52,5 \%(n=32)$ casado; $62,3 \%(n=38)$ del color blanco; 88,5\% ( $n=54)$ con antecedentes familiares de enfermedad crónica, siendo $63,9 \%$ $(n=39)$ con hipertensión arterial; $82 \%(n=50)$ con antecedentes personales, sumando 52,5\% $(n=32)$ de casos de infección urinaria; $70,5 \%(n=43)$ era multípara. El embarazo de riesgo evolucionó hasta cesariana en el $80,3 \%(n=49)$ de los casos. Este estudio produjo informaciones respecto al perfil de esta población, lo que permite a los profesionales involucrados en la atención a la embarazada de alto riesgo ejercer un papel fundamental en la reducción de la mortalidad materna. Se concluye que estas embarazadas de alto riesgo aparentemente no presentan perfil diferente de la realidad de otros municipios de Brasil, principalmente cuando la hipertensión fue constatada como factor importante de antecedente familiar, personal y prevalencia actual.

DESCRIPTORES: Embarazo de alto riesgo; Perfil de salud; Cuidado prenatal; Embarazadas; Salud de la mujer.

`Enfermeira. Mestre em Saúde e Gestão do Trabalho. Docente de Enfermagem da Universidade Paranaense. Francisco Beltrão, PR, Brasil.

${ }^{2}$ Enfermeira. Bacharel em Enfermagem. Universidade Paranaense. Francisco Beltrão, PR, Brasil.

${ }^{3}$ Enfermeiro. Mestre em Saúde e Gestão do Trabalho. Docente de Enfermagem da Universidade Paranaense. Francisco Beltrão, PR, Brasil.

${ }^{4}$ Nutricionista. Doutoranda em Nutrição. Docente da Universidade Paranaense. Francisco Beltrão, PR, Brasil.

${ }^{5}$ Educadora Física. Doutoranda em Ciências da Saúde. Docente da Universidade Paranaense. Francisco Beltrão, PR, Brasil.

Autor Correspondente:

Recebido: $02 / 12 / 2015$

Lediana Dalla Costa

Universidade Paranaense Unidade de Francisco Beltrão

Finalizado: 02/06/2016

R. São Francisco de Assis, 230 - 85604-180 - Francisco Beltrão, PR, Brasil

E-mail: lediana@unipar.br 


\section{- INTRODUÇÃO}

Há algumas décadas a saúde da mulher e da criança tem sido mundialmente estudada, e no Brasil é considerada prioridade, entretanto, o número de mortes decorrentes de complicações da gestação e do parto ainda é elevado ${ }^{(1-2)}$. Entre as diferentes complicações, destacam-se aqueles provenientes da gestação de alto risco.

A gestação de alto risco ocorre quando a gestante apresenta alguma doença ou condição sociobiológica como a hipertensão arterial, diabetes, alcoolismo, obesidade e outras, que prejudica a evolução da gravidez ${ }^{(3)}$, risco este que pode levar à morte materna.

No Brasil houve uma redução de 3,7\% da Razão da Mortalidade Materna (RMM) no entre os aos de 1990 a 2011. Apesar dessa redução, estudiosos salientam que os resultados não devem ser considerados animadores, visto que cada óbito materno precisa ser entendido como falha do sistema de saúde e como violação aos direitos humanos de reprodução ${ }^{(4)}$.

A partir dos dados do Sistema de Informação sobre Mortalidade (SIM) e do Sistema de Informação sobre Nascidos Vivos (Sinasc), com dados disponíveis no sítio do Departamento de Informática do Sistema Único de Saúde (Datasus), houve redução também na RMM no Paraná, visto que as taxas caíram de 51,7 em 2011, para 39 por 100.000 nascidos vivos em 2012, e os dados preliminares (ainda sujeitos a alteração) indicam para uma taxa próxima a 33,4 em 2014.

Recente estudo publicado demonstrou redução na RMM mundial entre os anos de 1990 a $2013^{(1)}$. Entretanto, apesar da importante redução da mortalidade materna, ainda há necessidade de ações de saúde pública e sociais mais efetivas ${ }^{(4)}$.

Nesse sentido, apesar do governo federal implementar a Rede Cegonha a partir da portaria $\mathrm{n}^{\circ}$ 1.459, de 24 de junho de $2011^{(5)}$ como forma de complementar o Programa de Humanização no Pré-natal e Nascimento (PHPN) ${ }^{(5-6)}$, o Estado do Paraná viu a necessidade de criar em 2013 o Programa Rede Mãe Paranaense, que consiste de um conjunto de ações para o acolhimento precoce da gestante, com acompanhamento pré-natal com no mínimo sete consultas, realização de 17 exames, classificação de risco das gestantes e das crianças, garantia de ambulatório especializado para as gestantes e crianças, e também do parto por meio de um sistema de vinculação ao hospital conforme o risco gestacional ${ }^{(7-8)}$.

De acordo com o Programa Rede Mãe Paranaense, a estratificação de risco para a gestante dividese em habitual, intermediário e alto risco. Esta classificação teve início após o estudo da Secretaria de Estado da Saúde do Paraná realizado para identificar mortalidade materna e infantil entre os anos de 2006 a 2010 no Estado, em que foram identificadas as principais causas de mortes e os fatores de risco que contribuem para a mortalidade materna e infantil ${ }^{(7)}$.

Diante do fato de que ainda é elevado número de gestações de alto risco, torna-se de suma importância a identificação dos fatores que levam à complicação de uma gravidez, a fim de verificar as possíveis medidas a serem adotadas para prevenção das complicações e reduções da RMM. Assim, traçar o perfil epidemiológico das gestantes de alto risco, atendidas em diferentes centros de referência regional, pode trazer informações importantes para desenvolver ações preventivas e corretivas imediatas para tais complicações.

Ressalta-se que na região do Sudoeste do Paraná há escassez de estudos epidemiológicos sobre gestação de alto risco. Vale destacar que a região é composta de municípios de pequeno e médio porte, localizados longe dos grandes centros, e carente de instituições de pesquisas com abordagem na área de obstetrícia, fato que pode dificultar a leitura da realidade local quanto aos fatores de risco gestacional. Assim, os resultados deste estudo poderão contribuir para identificar e sanar as dificuldades quanto à identificação precoce da gestação de alto risco na região, além de auxiliar nas práticas de promoção à saúde.

Portanto, o objetivo deste estudo foi traçar o perfil de gestantes de alto risco atendidas no Instituto da Mulher da Secretaria de Saúde do município de Francisco Beltrão, estado do Paraná. 
O presente estudo foi de caráter descritivo, quantitativo e documental retrospectivo, realizado no Instituto da Mulher da Secretaria de Saúde do Município de Francisco Beltrão, estado do Paraná (IMSSFB). Os dados foram obtidos em prontuários de primeira consulta puerperal junto ao IMSS-FB em todas as gestantes de alto risco $(n=61)$, que tiveram desfecho da gestação entre os períodos de janeiro a junho 2015.

O instrumento utilizado para a coleta de dados foi um questionário estruturado, elaborado pelos próprios autores a partir dos prontuários e das Fichas de Assistência Pré-natal (FAPN). As FAPN são uma padronização da Secretaria Municipal da Saúde do município de Francisco Beltrão e contêm informações sobre condições socioeconômicas; dos antecedentes familiares de primeiro grau (Hipertensão Arterial Sistêmica - HAS, Diabetes Mellitus - DM, malformação e gemelaridade); antecedentes pessoais e obstétricos; informações da gestação atual e exame físico.

A estratificação de risco para a gestante seguiu a Linha Guia do Programa Rede Mãe Paranaense da Secretaria de Saúde do Estado do Paraná(7), que considera as Condições Clínicas pré-existentes (Hipertensão arterial; Dependência de drogas lícitas e ilícitas; Cardiopatias; Pneumopatias; Nefropatias; Endocrinopatias; Hemopatias; Epilepsia; Doenças infecciosas; Doenças autoimunes; Ginecopatias; Neoplasias; Obesidade mórbida; Cirurgia bariátrica; Psicose e depressão grave) e as Intercorrências Clínicas (Doenças infectocontagiosas vividas durante a gestação atual - infecção de repetição do trato urinário, doenças do trato respiratório, rubéola, toxoplasmose, etc.; Doença hipertensiva específica da gestação; Doenças clínicas diagnosticadas pela primeira vez na gestação - cardiopatias, endocrinopatias; Retardo do crescimento intrauterino; Trabalho de parto prematuro; Placenta prévia; Amniorrexe prematura - abaixo de 37 semanas; Sangramento de origem uterina; Isoimunização Rh - Rh negativo); Má-formação fetal confirmada.

O diagnóstico quanto à doença de base que levou a gestante ao alto risco estava descrita na FAPN, com base na estratificação de risco descrita acima. Neste caso, não havia a apresentação do Código Internacional de Doença (CID). Além das informações acima, verificou-se o número de atendimentos, o tipo de parto predominante e o desfecho da gestação na primeira consulta puerperal.

Os prontuários foram analisados de forma anônima, sendo atribuída a numeração aleatória a fim de preservar o sigilo da identificação das gestantes.

Quanto aos critérios de inclusão, foram selecionados os prontuários das gestantes de alto risco no período do estudo e como critérios de exclusão foram os atendimentos das gestantes com pré-natal classificado como riscos habitual ou intermediário, além de outros atendimentos prestados durante o mesmo período pelo IMSS-FB, bem como as gestantes que não tiveram a evolução da gestação, por motivo de aborto no período do estudo.

Este estudo foi submetido e aprovado pelo Comitê de Ética e Pesquisa da Universidade Paranaense com parecer de aprovação número 1.047.397.

Para a análise dos dados, empregou-se estatística descritiva com análise de frequência para verificar as características gerais da amostra e dos diferentes riscos. Foi utilizado o programa de estatística SPSS (Statistical Package for Social Science), versão 17.0.

\section{RESULTADOS}

A partir da análise dos 61 prontuários deste estudo, verificou-se que a maioria das gestantes (82\%) tinha idade entre 15 e 35 anos $(n=50)$. Além disso, 36,1\% $(n=22)$ das gestantes foram identificadas como trabalhadoras do lar, 47,5\% ( $n=29)$ possuíam o segundo grau completo, $52,5 \%(n=32)$ eram casadas e $62,3 \%(n=38)$ de cor branca (Tabela 1$)$.

Dentre as gestantes analisadas, 54 mulheres (88,5\%) possuíam antecedentes familiares, sendo mais prevalente a Hipertensão Arterial Sistêmica (HAS) (63,9\%) verificada em 39 mulheres, seguido da gemelaridade (54,1\%) ( $\mathrm{n}=33)$ e da Diabetes Mellitus (DM) (34,4\%) ( $\mathrm{n}=21)$ (Tabela 2$)$. 
Verificou-se que $82 \%(\mathrm{n}=50)$ das mulheres possuíam antecedentes pessoais. A Infecção do Trato Urinário (ITU) foi estimada em 52,5\% $(n=32)$ das gestantes, seguido da HAS $(21,3 \%)(n=13)$. Vale ressaltar ainda que $52,4 \%(n=32)$ das gestantes apresentaram excesso de peso corporal, ou seja, sobrepeso e/ou obesidade, onde a obesidade isoladamente foi identificada em 18\% delas $(n=11)$. Em 36\% ( $n=22)$ dos casos havia dois ou mais antecedentes pessoais (Tabela 3 ).

Com relação aos antecedentes obstétricos das gestantes de alto risco analisadas a maioria (70,5\%) $(n=43)$ era multípara. Quanto ao tipo de parto atual resultante da gestação de alto risco, destacou-se o parto cesáreo, indicado em 80,3\% (n=49) dos casos atendidos pelo IMSS-FB (Tabela 4).

O principal motivo pelo qual a gestante participou do pré-natal de alto risco foi a HAS, diagnosticado em $24,6 \%(n=15)$ das gestantes, seguido de $18 \%(n=11)$ por obesidade e $14,8 \%(n=9)$ por ITU. Vale destacar que a mesma gestante pode apresentar mais do que uma patologia associada (Tabela 5).

Tabela 1 - Características demográficas das gestantes estratificadas como pré-natal de alto risco no Instituto da Mulher. Francisco Beltrão, Paraná, 2015

\begin{tabular}{lcc} 
Variáveis & $\mathbf{n}$ & $\%$ \\
\hline Classificação Idade & & \\
\hline $15-35$ anos & 50 & 82 \\
\hline Maior que 35 anos & 11 & 18 \\
\hline Profissão & & \\
\hline Agricultora & 4 & 6,6 \\
\hline Do Lar & 22 & 36,1 \\
\hline Auxiliar Administrativo & 4 & 6,6 \\
\hline Outros & 31 & 50,5 \\
\hline Escolaridade & & \\
\hline Nenhuma & 4 & 6,6 \\
\hline Primeiro Grau & 18 & 29,5 \\
\hline Segundo Grau & 29 & 47,5 \\
\hline Superior & 10 & 16,4 \\
\hline Estado Civil & & \\
\hline Casada & 32 & 52,5 \\
\hline Solteira sem união estável & 4 & 6,6 \\
\hline Solteira com união estável & 23 & 37,7 \\
\hline Separada & 2 & 3,3 \\
\hline Etnia & & \\
\hline Branca & 38 & 62,3 \\
\hline Negra & 3 & 4,9 \\
\hline Parda & 19 & 31,1 \\
\hline Ignorado & 1 & 1,6 \\
\hline & & \\
\hline & & \\
\hline
\end{tabular}

Tabela 2 - Antecedentes familiares das gestantes estratificadas como gestação de alto risco no Instituto da Mulher. Francisco Beltrão, Paraná, 2015

\begin{tabular}{lcc} 
Variáveis & $\begin{array}{c}\text { Sim } \\
\mathbf{n}(\mathbf{\%})\end{array}$ & $\begin{array}{c}\text { Não } \\
\mathbf{n}(\mathbf{\%})\end{array}$ \\
\hline Antecedentes familiares & & \\
\hline Hipertensão Arterial Sistêmica & $39(63,9)$ & $22(36,1)$ \\
\hline Diabetes Mellitus (DM) & $21(34,4)$ & $40(65,6)$ \\
\hline Malformação & $12(19,7)$ & $49(80,3)$ \\
\hline Gemelaridade & $33(54,1)$ & $28(45,9)$ \\
\hline Outros & $1(1,6)$ & $60(98,4)$ \\
\hline Quantidade de antecedentes familiares & \\
\hline Nenhum & $6(9,8)$ & $55(90,2)$ \\
\hline Um & $21(34,4)$ & $40(65,6)$ \\
\hline Dois & $18(29,5)$ & $43(70,5)$ \\
\hline Três & $13(21,3)$ & $48(78,7)$ \\
\hline Quatro & $3(4,9)$ & $58(95,1)$
\end{tabular}

Tabela 3 - Antecedentes pessoais das gestantes estratificadas como gestação de alto risco no Instituto da Mulher. Francisco Beltrão, Paraná, 2015

\begin{tabular}{lcc} 
Variáveis & $\begin{array}{c}\text { Sim } \\
\mathbf{n}(\mathbf{\%})\end{array}$ & $\begin{array}{c}\text { Não } \\
\mathbf{n}(\mathbf{\%})\end{array}$ \\
\hline Antecedentes pessoais & & \\
\hline Hipertensão Arterial Sistêmica & $13(21,3)$ & $74(8,7)$ \\
\hline Diabetes Mellitus & $1(1,6)$ & $60(98,4)$ \\
\hline Cirurgia Pélvica & $25(41)$ & $36(59)$ \\
\hline Cardiopatia & $2(3,3)$ & $59(96,7)$ \\
\hline Infecção do Trato Urinário & $32(52,5)$ & $29(47,5)$ \\
\hline Malformação & $3(4,9)$ & $58(95,1)$ \\
\hline Obesidade & $11(18)$ & $50(82)$ \\
\hline Outros & $5(8,2)$ & $56(91,8)$ \\
\hline Quantidade de antecedentes pessoais & \\
\hline Nenhum & $10(16,4)$ & $51(83,6)$ \\
\hline Um & $29(47,5)$ & $32(52,5)$ \\
\hline Dois & $15(24,6)$ & $46(75,4)$ \\
\hline Três & $6(9,8)$ & $55(90,2)$ \\
\hline Quatro & $1(1,6)$ & $60(98,4)$ \\
\hline
\end{tabular}


Tabela 4 - Antecedentes pessoais obstétricos das gestantes estratificadas como gestação de alto risco no Instituto da Mulher e o tipo de parto resultante da gestação atual. Francisco Beltrão, Paraná, 2015

\begin{tabular}{lcc} 
Variáveis & $\mathbf{n}$ & $\mathbf{\%}$ \\
\hline Nulípara & 18 & 29,5 \\
\hline Multípara & 43 & 70,5 \\
\hline Número de gestações & \\
\hline Gesta um & 18 & 29,5 \\
\hline Gesta dois & 16 & 26,2 \\
\hline Gesta três & 14 & 23 \\
\hline Gesta quatro ou mais & 13 & 21,3 \\
\hline Tipo de parto da gestação atual & & \\
\hline Parto Normal & 12 & 19,7 \\
\hline Parto Cesariana & 49 & 80,3
\end{tabular}

Tabela 5 - Doenças prevalentes nas mulheres estratificadas como gestação de alto risco no Instituto da Mulher. Francisco Beltrão, Paraná, 2015

\begin{tabular}{lcc} 
Variáveis & N & \% \\
\hline Hipertensão Arterial Sistêmica & 15 & 24,6 \\
\hline Dependência de drogas & 2 & 3,3 \\
\hline Cardiopatia & 1 & 1,6 \\
\hline Pneumopatia & 1 & 1,6 \\
\hline Nefropatia & 1 & 1,6 \\
\hline Endócrino (Diabetes & Mellitus, & 8,2 \\
tireoide, etc.) & & \\
\hline Hemopatias & 6 & 9,8 \\
\hline Doenças infecciosas & 5 & 8,2 \\
\hline Doença autoimune & 1 & 1,6 \\
\hline Ginecopatia & 3 & 4,9 \\
\hline Obesidade & 11 & 18 \\
\hline Psicose, Depressão & 5 & 8,2 \\
\hline Patologia do feto & 8 & 13,1 \\
\hline Cirurgia pélvica & 1 & 1,6 \\
\hline Gemelaridade & 2 & 3,3 \\
\hline Infecção do trato urinário & 9 & 14,8 \\
\hline Outras causas & 22 & 36,1 \\
\hline Total de ocorrências & 98 & -- \\
\hline & &
\end{tabular}

\section{- DISCUSSÃO}

Este estudo analisou o perfil de 61 gestantes de alto risco acompanhadas no IMSS-FB. Vale lembrar que a gestação de alto risco é determinada a partir de algumas condições clínicas preexistentes, das doenças clínicas diagnosticadas pela primeira vez na gestação, bem como das intercorrências clínicas ${ }^{(7-9)}$.

Em sua maioria as gestantes eram mulheres jovens, em idade fértil, com predomínio da faixa etária de 15 a 35 anos (82\%). Essa informação diverge de estudo realizado no estado do Pará(10), em um centro de referência em pré-natal de alto risco de hospital regional, que avaliou prontuários de gestantes de alto risco e encontrou 43 casos com idade entre 16 a 25 anos. Assim, a idade aparentemente não foi um fator determinante para o risco gestacional desse estudo. Isto posto, a literatura relata que um risco aumentado é observado nas gestações ocorridas na fase adolescente, menor que 15 anos, bem como para mulheres com idade acima de $35 \operatorname{anos}^{(9)}$.

Quanto à escolaridade, observou-se que 47,5\% das gestantes apresentaram o ensino fundamental completo. Em estudo realizado no sul do estado de Santa Catarina, realizado com prontuários de 1510 gestantes entre os anos de 2012 e 2013, foi verificado que 23,9\% das gestantes possuíam o ensino fundamental incompleto ${ }^{(11)}$. O mesmo estudo identificou ainda que 52,8\% das gestantes tinham união estável, enquanto as casadas eram em menor proporção (34\%). Nesta pesquisa os dados aparecem divergentes, mais gestantes casadas, seguidas daquelas com união estável.

Quase todas as características das gestantes desta pesquisa se aproximam de estudo realizado no Brasil entre os anos de 2011 e $2012^{(12)}$. Os autores identificaram características demográficas de 23.894 puérperas: 70,4\% tinham idade entre 20 a 34 anos, 56,8\% eram pardas, 26,1\% possuíam até oito anos de escolaridade, $85,5 \%$ viviam com o companheiro e 52,7\% eram multípara. Destacam ainda que abaixa adequação da assistência pré-natal, importantes desigualdades e proporção inadequada de assistência podem ser observadas em mulheres com menos escolaridade, classe econômica mais baixa, multíparas e sem companheiro. 
De acordo com o Manual Técnico da Gestação de Alto Risco ${ }^{(9)}$, a baixa escolaridade pode representar um fator de risco principalmente porque está relacionada ao menor acesso à informação e ao limitado entendimento da importância dos cuidados com a saúde.

Outro fator analisado foi a etnia. Verificou-se que a cor branca predominou em $62,3 \%$ dos casos. Em estudo realizado no Centro de Atendimento à Mulher do interior do Mato Grosso do Sul, considerado de referência para o pré-natal de risco ${ }^{(13)}$, verificou que $63,89 \%$ das gestantes eram da cor branca. Estes resultados não estão de acordo com a literatura que indica que a raça não branca pode representar um fator de risco para síndromes hipertensivas em gestantes, e representa uma das principais patologias obstétricas entre as gestantes de risco $^{(14)}$.

Os antecedentes familiares representam um fator importante na classificação do risco da gestante. O presente estudo identificou que $88,5 \%$ delas possuíam algum antecedente familiar de doenças crônicas, contudo, destaca-se como agravante a combinação de dois ou mais antecedentes familiares. Estudo ${ }^{(8)}$ destaca que a somatória de antecedentes familiares ou pessoais pode aumentar a probabilidade de agravo na gestação.

O antecedente familiar mais prevalente foi a HAS apresentado em $63,9 \%$ dos casos. Resultados semelhantes $(62,5 \%)$ foram encontrados no estudo realizado em uma unidade de internação de Ginecologia e Obstetrícia de Hospital Geral e Maternidade do Sistema de Saúde de Fortaleza ${ }^{(15)}$, referência terciária para gestação de alto risco, do qual participaram 40 gestantes no período de janeiro a fevereiro de 2006.

Outro fator agravante para gestação de risco encontrado neste estudo foi os antecedentes pessoais, ou seja, a presença prévia de doenças crônicas ou infecções recorrentes, sendo a ITU a mais evidente nos prontuários analisados $(52,5 \%)$. O risco da ITU na gestação foi relatado em estudo ${ }^{(16)}$ como fator relevante para complicação do período gestacional, visto que agrava tanto o prognóstico materno quanto o perinatal. E neste caso observou-se ainda que, durante a gestação, $14,8 \%$ das mulheres desenvolveram ITU.

Quanto ao histórico obstétrico dos prontuários analisados, observou-se que a maioria (70,5\%) das mulheres eram multíparas, com $23 \%$ dos casos na terceira e $21,3 \%$ na quarta gestação. Além disso, o risco gestacional das mulheres deste estudo pode ter levado à prevalência de $80,3 \%$ de parto cesáreo, encontrado neste estudo.

Em estudo realizado com 94 gestantes em um centro de referência em pré-natal de alto risco do Hospital Regional do Baixo Amazonas do Pará(10), durante o período de um ano (2010-2011), dentre 58 gestantes $67 \%$ resultaram em parto cesáreo, enquanto, 33\% tiveram seus filhos por parto normal.

O manual técnico de Gestação de Alto Risco do Ministério da Saúde ${ }^{(9)}$ recomenda que a cesárea eletiva seja uma opção em situações de iteratividade, ou seja, duas ou mais cicatrizes anteriores e em casos de contraindicação absoluta ao parto vaginal, excetuando-se as situações de urgência e sofrimento fetal.

Em relação à causa que caracterizou o pré-natal de alto risco das gestantes, verificou-se que a HAS foi responsável por 15 encaminhamentos, isto é, mulheres portadoras de HAS crônica ou as que desenvolveram Doença Hipertensiva Específica da Gestação (DHEG). A hipertensão arterial gestacional é considerada uma das mais importantes complicações do ciclo gravídico puerperal, resultando em alto risco de morbidade e mortalidade materna e perinatal ${ }^{(17)}$.

Os distúrbios hipertensivos são as complicações mais comuns no pré-natal, acometendo 12 a $22 \%$ das gestações, sendo a eclâmpsia uma das principais causas de óbito materno em países desenvolvidos e em desenvolvimento ${ }^{(15)}$.

O segundo maior fator de direcionamento para o alto risco foi a obesidade, responsável por 11 casos. Em estudo realizado com 164 gestantes de uma maternidade pública de alto risco, em Goiânia, estado de Goiás ${ }^{(18)}$, no período de janeiro a julho de 2012, 47,8\% gestantes apresentavam excesso de peso pré-gestacional, sendo que 48,5\% apresentaram estado nutricional adequado.

Em relação ao estado nutricional durante a gestação, mais da metade (52,4\%) das gestantes apresentaram excesso de peso. Vale destacar que a obesidade materna e o ganho de peso ponderal 
elevam os riscos para diabetes gestacional, parto prolongado, pré-eclâmpsia, cesárea e depressão. Para o recém-nascido, observa-se maior morbidade neonatal e maior probabilidade de obesidade, sobrepeso e distúrbios metabólicos na infância e adolescência ${ }^{(19)}$.

Diante desse quadro de gestação de risco, a literatura destaca que o acompanhamento pré-natal é essencial para garantir a gestação segura e/ou saudável, além de parto seguro e esclarecimentos de dúvidas das futuras mães ${ }^{(20)}$. As gestantes que recebem assistência de qualidade no pré-natal, com eficiência e eficácia em suas ações, têm possibilidade de redução de morbidades que podem persistir mesmo após o término da gestação, como também evitar mortalidade materna, baixo peso do recémnascido, retardo do crescimento intrauterino, entre outras complicações ${ }^{(2)}$.

Durante o acompanhamento pré-natal, a gestante recebe informações sobre cuidados importantes, tanto para saúde do bebê quanto da mãe, tais como aleitamento materno, alimentação balanceada, prática de exercícios físicos, orientação sobre vacinação, além de exames laboratoriais e de imagem ${ }^{(9,12,21)}$.

Os resultados do presente estudo podem ser relevantes, principalmente porque evidência que a HAS, obesidade e ITU relatada pela literatura, são importantes fatores de agravo do risco às gestantes. Assim, ações de acompanhamento e cuidados pré-natais são primordiais nesta população.

\section{CONSIDERAÇÕES FINAIS}

Este estudo permitiu constatar que as gestantes do município de Francisco Beltrão, estado do Paraná, que participam no pré-natal de alto risco, aparentemente não apresentam perfil diferente da realidade de outros municípios do Brasil, principalmente quando identifica-se que a HAS foi constatada como importante fator de antecedente familiar, pessoal e prevalência atual. A ITU e a obesidade foram encontrados e podem levar a complicações na gestação.

Destaca-se a importância de pré-natal qualificado, com especial atenção ao histórico familiar e pessoal da gestante, além de um número e tempo de atendimentos adequados para as consultas e a realização dos exames em cada trimestre, visando identificar previamente qualquer alteração no desenvolvimento da gestação.

Apesar de o presente estudo ter proporcionado informações importantes relacionadas à saúde da gestante, uma limitação deve ser considerada. A maioria das pesquisas recentes, com abordagem na área de obstetrícia de alto risco da população brasileira, é concentrada nas regiões Norte e Nordeste do País. Com isso, os resultados encontrados no presente estudo limitam algumas comparações com as diferentes regiões do país. Assim, em estudos futuros, quando realizadas as comparação entre os fatores para a gestação de alto risco, devem-se considerar os fatores econômicos, sociais e culturais entre as diferentes regiões analisadas.

\section{REFERÊNCIAS}

1. GBD 2013 Risk Factors Collaborators, Forouzanfar MH, Alexander L, Anderson HR, Bachman VF, Biryukov S, et al. Global, regional, and national comparative risk assessment of 79 behavioral, environmental and occupational, and metabolic risks or clusters of risks in 188 countries, 1990-2013: a systematic analysis for the Global Burden of Disease Study 2013. The Lancet. 2015; 386(10010): 2287-2323.

2. Peixoto CR, Freitas LV, Teles LMR, Campos FC, de Paula PF, Damasceno AKC. O pré-natal na atenção primária: o ponto de partida para reorganização da assistência obstétrica. Rev. Enferm. UERJ. 2011; 19(2): $286-91$.

3. Luz BG, Soares LT, Grillo VTRS, Viola MB, Laporte IC, Bino DBM, et al. O perfil das gestantes de alto risco acompanhadas no pré-natal da policlínica de Divinópolis-MG, no biênio 2013-14. J. Health Biol. Sci. 2015; 3(3): 137-43.

4. Szwarcwald CL, Escalante JJC, Rabello Neto DL, de Souza Junior PRB, Victora CG. Estimação da razão de mortalidade materna no Brasil, 2008-2011. Cad. Saúde Pública. 2014; 30(Suppl 1): S71-S83.

5. Brasil. Portaria n. 1.459/GM/MS, de 24 de junho de 2011: Institui, no âmbito do Sistema Único de Saúde - SUS 
- a Rede Cegonha. Brasília: Ministério da Saúde; 2011.

6. Martinelli KG, dos Santos Neto ET, da Gama SGN, Oliveira AE. Adequação do processo da assistência prénatal segundo os critérios do Programa de Humanização do Pré-natal e Nascimento e Rede Cegonha. Rev. Bras. Ginecol. Obstet. 2014; 36(2): 56-64.

7. Paraná. Secretaria de Estado da Saúde do Paraná. Superintendência de Atenção à Saúde. Linha Guia da Rede Mãe Paranaense. Paraná: Secretaria de Estado da Saúde do Paraná; 2013.

8. Huçulak MC, Peterlini OLG. Rede Mãe Paranaense - Relato de experiência. Revista Espaço para a Saúde. 2014; 15(1): 77-86.

9. Brasil. Ministério da Saúde. Secretaria de Atenção à Saúde. Departamento de Ações Programáticas Estratégicas. Gestação de alto risco: manual técnico. $5^{\mathrm{a}}$ ed. Brasília: Editora do Ministério da Saúde; 2012.

10. dos Anjos JCS, Pereira RR, Ferreira PRC, Mesquita TBP, Picanço Júnior OM. Perfil epidemiológico das gestantes atendidas em um centro de referência em pré natal de alto risco. Rev. Para. Med. 2014; 28(2): 23-33.

11. Rosa MRQP, Silva MS. Perfil de gestantes de alto risco atendidas em um centro obstétrico de Santa Catarina. R. Interd. 2014; 7(2): 95-102.

12. Domingues RMSM, Viellas EF, Dias MAB, Torres JA, Theme-Filha MM, da Gama SGN, et al. Adequação da assistência pré-natal segundo as características maternas no Brasil. Rev Panam Salud Publica. 2015; 37(3): 140-7.

13. Rezende CL, Souza JC. Qualidade de vida das gestantes de alto risco de um centro de atendimento à mulher. Psicol. inf. 2012; 16(16): 45-69.

14. Teixeira LA, Vasconcelos LD, Ribeiro RAF. Prevalências de Patologia e Relação com a Prematuridade em Gestação de Alto Risco. Rev. cienc. saude. 2015; 5(4): 1-8.

15. Moura ERF, de Oliveira CGS, Damasceno AKC, Pereira MMQ. Fatores de risco para síndrome hipertensiva específica da gestação entre mulheres hospitalizadas com pré-eclâmpsia. Cogitare Enferm. 2010; 15 (2): 250-5.

16. Hackenhaar AA, Albernaz EP, Tomasi E. Infecção urinária sintomática na gestação e sua associação com desfechos neonatais e maternos desfavoráveis. Vittalle. 2011; 23(2): 19-26.

17. Martins M, Monticelli M, Brüggemann OM, Costa R. A produção de conhecimento sobre hipertensão gestacional na pós-graduação stricto sensu da enfermagem brasileira. Rev. esc. enferm. USP. 2012; 46(4): 802-8.

18. Godinho JCM, Rezio MA, da Silva LP, Freitas ATV, Martins KA, do Amaral WN. Ganho ponderal excessivo em gestantes atendidas em serviço público de alto risco. Fragm. Cult. 2014; 24(n.esp.): 85-95.

19. Nomura RMY, Paiva LV, Costa VN, Liao AW, Zugaib M. Influência do estado nutricional materno, ganho de peso e consumo energético sobre o crescimento fetal, em gestações de alto risco. Rev. Bras. Ginecol. Obstet. 2012; 34(3): 107-12.

20. Morse ML, Fonseca SC, Barbosa MD, Calil MB, Eyer FPC. Mortalidade materna no Brasil: o que mostra a produção científica nos últimos 30 anos? Cad. Saúde Pública. 2013; 27(4): 623-38.

21. Brasil. Ministério da Saúde. Secretaria de Atenção à Saúde. Departamento de Atenção Básica. Atenção ao prénatal de baixo risco. Brasília: Editora do Ministério da Saúde; 2012. 